\title{
Dynamic Neighbourhood Cellular Automata
}

\author{
Stefan Dantchev \\ Department of Computer Science, Durham University \\ South Road, Durham, Co. Durham, DH1 3LE, UK \\ s.s.dantchev@durham.ac.uk
}

\begin{abstract}
We propose a definition of Cellular Automaton in which links between cells can change during the computation. This is done locally by each cell, which can reach the neighbours of its neighbours in a single computational step. We suggest that Dynamic Neighbourhood Cellular Automata can serve as a theoretical model for studying Algorithmic and Computational Complexity issues in the are of Ubiquitous Computing. We illustrate this approach by giving an optimal logarithmic time solution of the Firing Squad Synchronisation problem in our model, which is an exponential speed-up over classical Cellular Automata.
\end{abstract}

Keywords: Cellular Automata, Ubiquitous Computations, Algorithms, Computational Complexity

\section{INTRODUCTION}

General background The concept of Cellular Automaton (CA) goes back to works by Von Neumann and Ulam in the early 1950's [7]. A CA is a discrete structure consisting of identical cells arranged in a regular way (such as a line, a two dimensional square grid, etc.). Each cell is a deterministic finite-state automaton (DFA) whose transition function depends on its own state as well as on the states of the neighbours, and the cells' states change synchronously in a single computational step. Thus, one can think of a CA as a massively parallel system, which consists of very simple building blocks than can interact only locally. Von Neumann himself was motivated by his own question of existence of self-reproducing machines, and proved that there is a CA that can perform a universal computation. Since then, CA have received much attention not only from theoretical point of view but also have been used in practice for modelling and simulations [3]. It is now known that even very simple one-dimensional CA can exhibit a very complex global behaviour [11], and therefore issues such as reversibility, conservation laws, limit sets, decidability questions, universality and topological dynamics of CA have been extensively studied [4].

Motivation and Results In our paper, we focus on Algorithmic and Computational Complexity aspect of a certain less-studied kind of CA, namely one in which a cell can dynamically change its neighbourhood by linking to neighbours of neighbours. One can argue that such a model, which we call Dynamic Neighbourhood Cellular Automaton (DNCA), can serve as a basis for studying computational resources in Ubiquitous Computing - a new, quickly developing area of Computer Science, which is one of the UKCRC grand challenges [10]. To illustrate the advantages of DNCA over classical static-neighbourhood CA, we show an exponential speed-up of the former over the latter using the classical Firing Squad Synchronisation Problem (FSSP). More precisely, we give an algorithm that solves it in optimal $\Theta(\log n)$ time on a DNCA consisting of $n$ soldiers.

Related work It seems that Rosenfeld and Wu were the first to consider CA that can dynamically change links between cells. While earlier work [8] was on recognition of certain regular structures of the underlying graph, the subsequent paper [9] contains several algorithms for transforming a regular link structure to another. The presentation of [9] is however rather informal. A more 
serious drawback, in our opinion, is that the authors did not recognise the importance of the potential speed-up of CA with dynamic links over classical ones. For instance, they presented a linear (in the number of cells) algorithm for converting a line into a balanced binary tree. In this paper, we show that this can be done in logarithmic time, which is optimal up to a constant factor. As a matter of fact, Rosenfeld and Wu used the FSSP as a subroutine in their construction. We have had different motivation and quite the opposite approach - we suggest the use of creating a balanced binary tree as a building block for faster computations, and in particular for solving the FSSP problem in logarithmic time.

More recently, Dubacq [2] reconsidered the dynamic neighbourhood in the context of CA and compared several different models. He also proved a quite general "synchronisation" theorem, which included solving the FSSP in logarithmic time. While Dubacq's dynamically reconfigurable $\mathrm{CA}$ have been rigorously defined and the importance of the the speed-up (logarithmic versus linear) have been acknowledged, his model has a serious drawback in that it allows for a cell to be seen by an unbounded number of other cells. Firstly, this does not look reasonable from a practical point of view that a processor can send information to everyone else in a single computational step. Secondly, it allows for a straightforward solution of the FSSP by simply pointing everyone to the general - this can trivially be done in logarithmic time. In contrast to this, our definition bounds the number of connections, which a cell can have at any time, by a constant (the same holds in the model of Rosenfeld and $\mathrm{Wu}$ ).

Finally, it is worth mentioning some related sequential computational models (related in that they have the notion of changing links in their memory) that are called pointer machines or storagemodification machines (see the survey [1]).

Rest of the paper is organised as follows. In section 2, we give the formal definition of what we call Dynamic-Neighbourhood Cellular Automata (DNCA). We have tried to keep it as simple as possible yet general enough so that it can be easily extended in different ways, which we discuss in section 4. The proof of the main result, a solution of the FSSP in $\Theta(\log n)$ time, is given in section 3 . Finally, we discuss possible directions for further research in section 4 .

\section{DEFINITIONS}

The formal definition of DNCA and some related concepts is as follows.

\section{Definition 1}

DNCA is a quadruple $(Q, P, \delta, \mathcal{C})$ where

1. $Q$ is a finite set of states;

2. $P$ is a finite sets of ports;

3. $\delta: Q^{|P|+1} \rightarrow Q \times\left(\{\varepsilon\} \cup P \cup P^{2}\right)^{|P|}$ is the transition function and

4. $\mathcal{C}$ is a (potentially infinite) set of cells.

An interconnection function is a function $\eta: \mathcal{C} \times P \rightarrow \mathcal{C} \times P \cup\{\perp\}$ such that $\eta(\eta(C, p))=\langle C, p\rangle$ for every $C \in \mathcal{C}$ and $p \in P$ such that $\eta(C, p) \neq \perp$.

A global state of the DNCA is a pair $\left(Q^{|\mathcal{C}|}, \eta\right)$.

The intuitive meaning of the definition is as follows. We have a set of identical Deterministic Finitestate Automata (DFAs) that we call cells. The cells are "named" by the elements of some set $\mathcal{C}$. Usually, we take $\mathcal{C}$ to be some countable set, e.g. the natural numbers $\mathbb{N}$. In the rest of the paper, however, we shall be mainly concerned with time complexity and, thus, we shall assume than only finitely many cells numbered from 1 to $n$ are active - here $n$ plays the role of "input size". We note that the cell names have no relevance to the actual computation of a DNCA as a cell is a DFA, which cannot hold $\log n$ bits required to memorise a name. All the cells have a common 
state set $Q$ and the same transition function $\delta$ which depends on the cell's own state as well as on the states of up to $|P|$ neighbouring cells which are available through the cell's ports named by elements of some finite set $P$.

The links are formally defined with the help of the interconnection function: if two cells $C_{1}$ and $C_{2}$ are connected through ports $p_{1}$ of $C_{1}$ and $p_{2}$ of $C_{2}$, the interconnection function should consistently say so, i.e. $\eta\left(C_{1}, p_{1}\right)=\left\langle C_{2}, p_{2}\right\rangle$ and $\eta\left(C_{2}, p_{2}\right)=\left\langle C_{1}, p_{1}\right\rangle$. A port $p$ of a cell $C$ may be left loose - this is reflected by $\eta(C, p)=\perp$ and may be used to define external input/output to the DNCA (however, we ignore this issue throughout the paper). Note that the definition allows for loops, i.e. $\eta(C, p)=\langle C, p\rangle$.

The transition function $\delta$ takes the current state of a cell $C$ together with the current states of its (at most) $|P|$ neighbours and then returns a new state plus $|P|$ port changes, each of them being one of the following three types: $\varepsilon$ (leave the port loose), $p$ (connect to the cell which is currently connected to the port $p$ of $C$ ) or $\left\langle p_{1}, p_{2}\right\rangle$ (connect to the cell which is currently connected to port $p_{2}$ of the cell which is connected to port $p_{1}$ of $C$ - i.e. switch a connection from a neighbour to a neighbour of a neighbour). All these changes that produce a new global state from the current one happen synchronously in parallel. It could happen that the new interconnection function is inconsistent, i.e. $\eta(C, p) \neq \perp$ and $\eta(\eta(C, p)) \neq\langle C, p\rangle$ for some $C \in \mathcal{C}$ and $p \in P$ - we shall treat such a situation as run-time error, in which case the computation of the DNCA fails.

\section{FIRING SQUAD IN OPTIMAL LOGARITHMIC TIME}

Firing Squad Synchronisation Problem is a synchronisation problem for a line of finite automata (i.e. one-dimensional CA) proposed by Myhill in 1957, first solved by McCarthy and Minsky, and appeared in print in [6]. The problem statement is as follows. The first (leftmost) cell is a general and all the others are soldiers. At some point in time, the general is placed in a special state "fire when ready". The task is to turn all soldiers into a "fire" state simultaneously at some later time, and it must be the first time that any of them has fired.

The simple divide-and-conquer solution of Moore starts by the general sending two signals along the line: a fast signal and a slow one, which moves three times as slow. The fast signal bounces off the end of the line and meets the slow signal in the centre. The middle soldier declares himself a general and with the help of additional two signals, he agrees with the original general that they both give order to fire at the same time, each for his half of the line. Thus, the process recursively continues, halving each subline until each division consists of a single soldier (who becomes general at that moment). Then every soldier fires. It is easy to see that the time required is $O(n)$. A number of other solutions have been found including an absolute optimal ones in terms of time, number of states, communication bits etc. The problem has been generalised to many different topologies (see the survey [5]).

In order to formalise the FSSP, we shall assume throughout the section that we are given a DNCA with $n$ linearly ordered cells. That is, the cell names are the numbers from 1 to $n$, and there are we successor and predecessor ports defined by $\operatorname{succ}(x)=x+1$ for every $x \in[1 \ldots n-1]$, $\operatorname{succ}(n)=\varepsilon$ and pred $(x)=x-1$ for every $x \in[2 \ldots n]$, pred $(1)=\varepsilon$. We also assume that, in the beginning, all the cells are in some idle state except for cell 1 , which initiates the computation, and cell $n$, which is in an end state, i.e. knows that it is the last in the line.

Our idea is to convert the line into a balanced binary tree (BBT) and then run a classical FSSP algorithm (i.e. any one that solves the problem on a static-neighbourhood $C A$ ) simultaneously on all branches (paths from the root to a leaf). Since the depth of a BBT of $n$ nodes is roughly $\log n$, we will be done if we can create the tree in time $O(\log n)$. Recall that an algorithm for transforming a line into a BBT was given [9] but it uses a classical FSSP algorithm running on lines of length $n$ initially, thus takes time $O(n)$. 
Creating the BBT is done at two stages. The first one produces an almost balanced binary tree (ABBT), and the second one balances it by identifying the leaves that are a level higher than the other leaves.

Building the ABBT is done recursively, by setting the root to be cell 1 and then recursively constructing two disjoint almost balanced subtrees - one with a root 2 and containing all evennumbered cells, and the other with root 3 and containing all odd-numbered cell (except 1, of course). The recursive split is made in constant time, by simply linking any cell whose name is $\geq 4$ to the neighbour of the neighbour in both directions. Special care is needed in the boundary cases, i.e. the cells that are neighbours of the end(s) or the root(s).

More formally, the first stage is meant to leave any cell in a state of the form (vertex-type, parent-type). Here vertex-type is leaf (a leaf of the tree), lean (a vertex that has a single left child which is a leaf) or node (any other internal vertex, including the root). parent-type is left, right (the node is a left, respectively right, child) or none (for the only root of the tree). The process starts by cell 1 going to a state (root, none). A root node links the next two nodes as the left and the right (only if it exists) children:

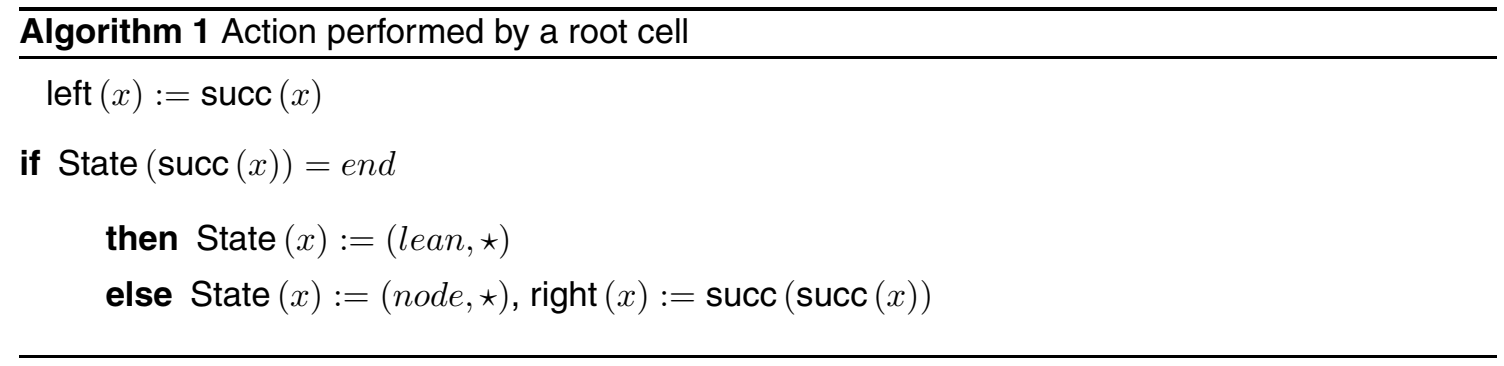

(Here and thereafter, the $\star$ denotes a part of the state, which we do not care about and do not change subsequently.) An idle cell should become a left/right root only if its predecessor/predecessor of predecessor has been reached. Otherwise it should link to the neighbour of the neighbour in both directions with special care needed if it is next to an end cell:

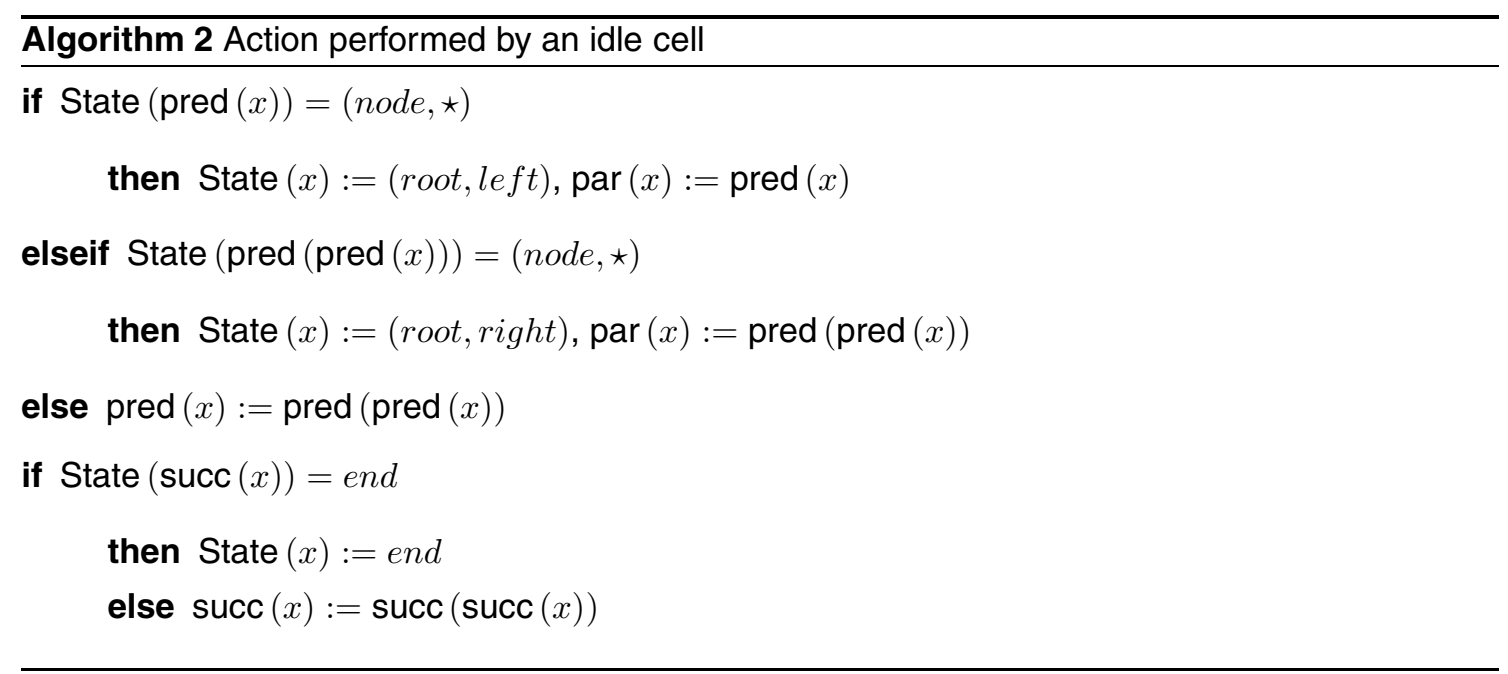

An end cell should become a left/right leaf only if its predecessor/predecessor of predecessor has been reached: 


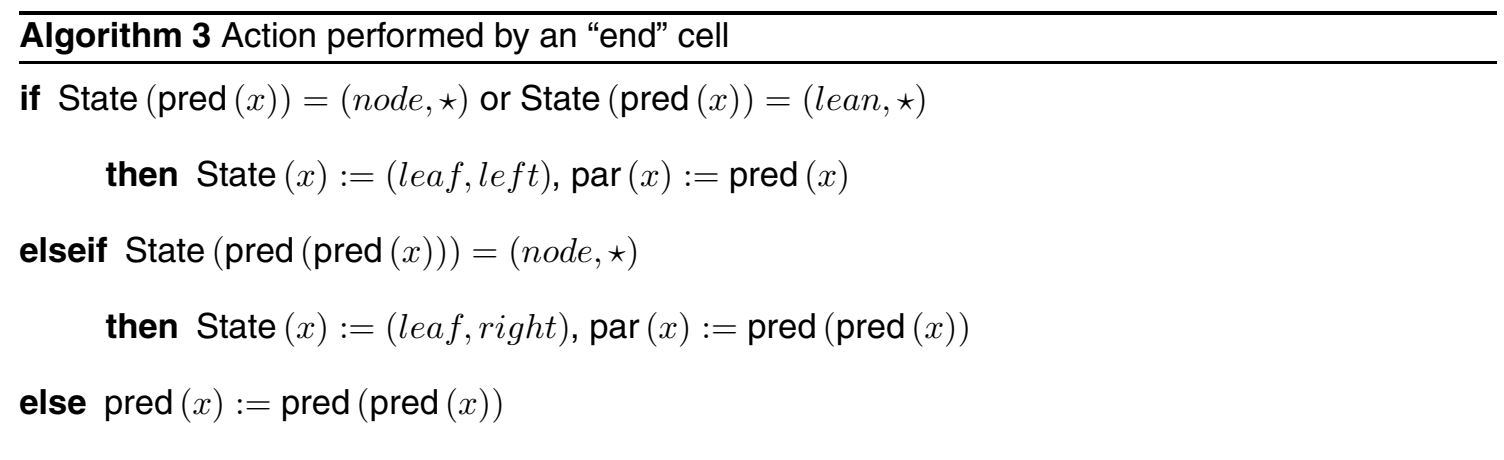

The figure below is an example of how the first stage works on a 8-element line. The doublecircled vertices show the recursive propagation of the root(s). In the end, 1,2, 3 are nodes, 4 is a lean, and $5,6,7,8$ are leaves.
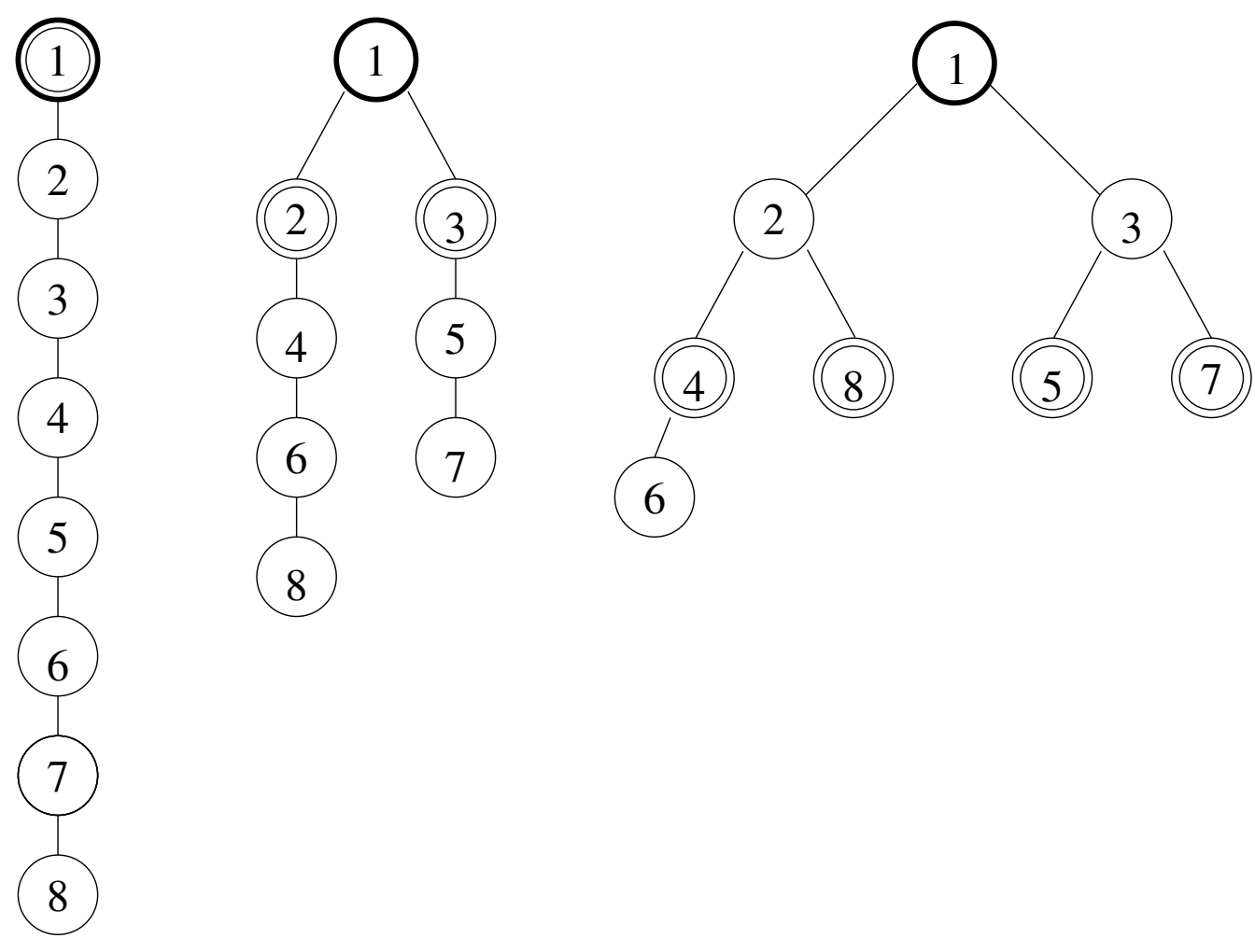

8

Figure 1: Creating an almost BBT.

One can easily prove, by induction on the number of nodes, the following.

Proposition 2 The ABBT, produced by the algorithm above, has the following properties.

1. The height of the tree is $h=\left\lfloor\log _{2} n\right\rfloor+1$.

2. For every internal node $v$, the difference between the sizes (number of nodes) of the left subtree and the right subtree rooted at $v$ is either zero or one.

3. Every leaf of the tree is at depth either $h$ or $h-1$.

The purpose of the second stage is 
Balancing the ABBT This is done by finding all the leaves at the higher depth $h-1$ and advising them to pretend that they have a fake child (at level $h$ ) - we call such leaves extended. This could be easily achieved recursively. Given a node with left and right subtrees $L$ and $R$, respectively, the height of $L$ is greater than the height of $R$ if only if $R$ is perfectly balanced and $L$ has a node more than $R$, i.e. $|L|=|R|+1=2^{t}$ for some $t$. In this case all leaves of $R$ should be extended and $L$ should be recursively balanced - this is the base case of the recursion. Otherwise, $L$ and $R$ have the same height, so they should be simultaneously balanced.

The implementation of this stage is better described in terms of message passing. It is initiated by the unique root of almost BBT who sends a message balance to both his children. This message is passed down by ordinary nodes until it reaches a lean or a leaf. A lean is a root of subtree of size two, so it replies back to its parent by a message power whose informal meaning is "my subtree is of size which is a perfect power of two". A leaf replies back to its parent by a message balanced whose meaning is "my subtree is of perfectly balanced", i.e. of size which is a perfect power of two minus one. Now every node awaits messages from both children. Whenever a node receives two balanced, it passes balanced to its parent. If a node gets power from the left and balanced from the right, every leaf of the right subtree should be extended. This is done by sending two message at the same time, an extend to the right child and a power to the parent, respectively (the latter message reflects the fact that the size of the subtree is a perfect power of two $2^{t+1}$ if and only if the left subtree is of size $2^{t}$ while the right one is of size $2^{t}-1$ and, thus, balanced). Any other combination of two messages from the children can simply be ignored as the subtree rooted at the current node is neither balanced nor is its size a perfect power of two. Any extend message is passed down until it reaches a leaf, which then gets to know that it should behave as it had a child.

An example with 18 nodes is given below. The black nodes are roots of balanced subtrees, while the grey ones are roots of subtrees whose size is a perfect power of two. The grey node vertices $3,4,5,6$ sent extend message to all leaves in their respective right subtrees.

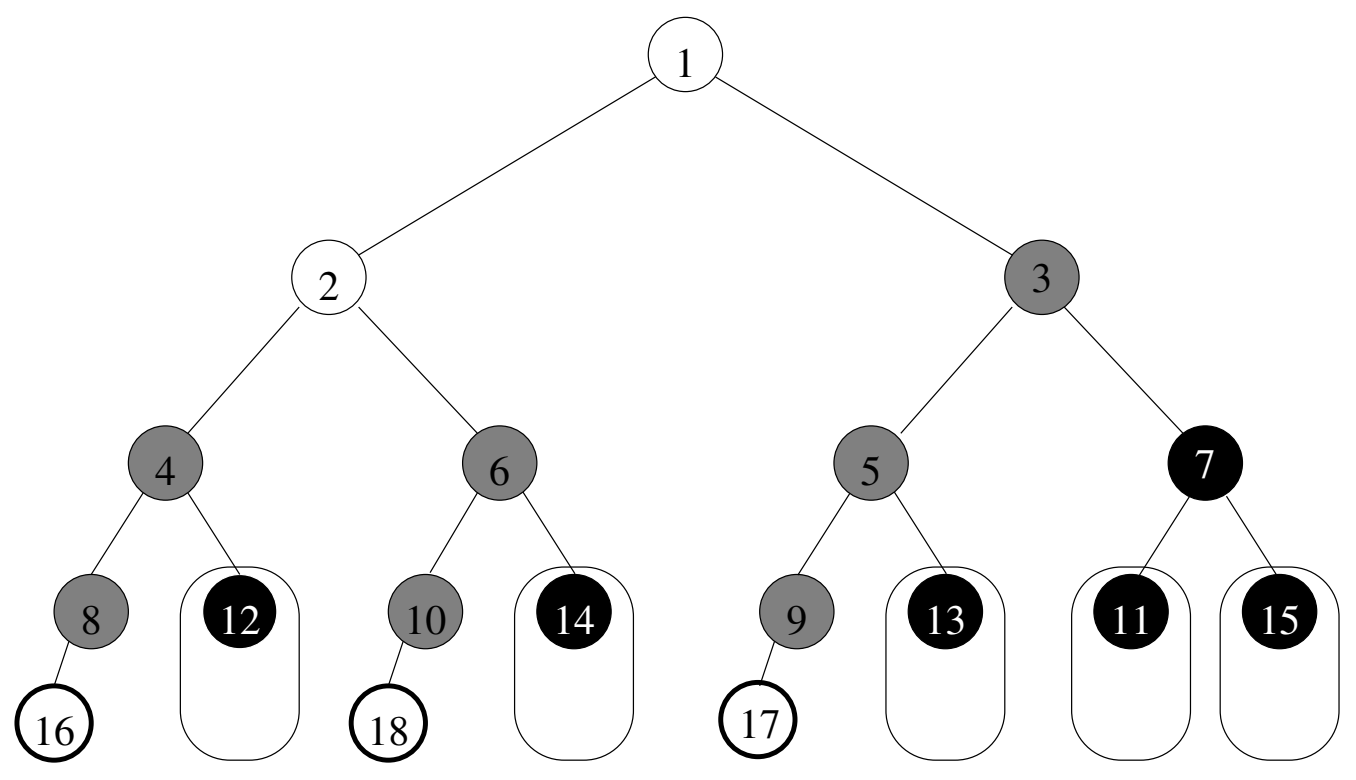

Figure 2: Completing the tree

There is a final bit, which is not actually needed for solving the FSSP, but may be needed in other applications that use the conversion to a BBT, so we briefly describe it. This is letting the root know that the algorithm has finished, i.e. every leaf knows if it is extended or not and, thus, will behave accordingly in the future. At first, it seems that this could be done by every leaf and lean sending 
a message ready back to their parents, and by every internal node sending awaiting for two ready messages (from both children) and the passing it up. This does not work as a some leaves may find out at a later stage that they should be extended. The problem is easily fixed by observing that any incorrect ready message (going up) will eventually meet an extend message going down - in such a case the ready message(s) is/are simply cancelled. A leaf, which is extended, then has to send a second ready message that will not be cancelled and thus every node will eventually get two correct ready messages.

As for

Solving the FSSP we point out that the two stages of creating the BBT can work in parallel (more precisely the second stage lags a step behind the first one) - it is not hard to see that whenever a node needs to send or receive a message, the relevant links as well as the type of the node has already been established by the first stage. In order to solve the FSSP, we can run any static-neighbourhood algorithm on all branches in parallel with a speed three times slower than normal - it is easy to see that every signal sent by such an algorithm will reach a soldier who has already established his position (i.e. knows he is the end of the line, or knows he has to simulate an additional fake soldier). The running time is clearly $O(\log n)$. The optimality follows from the fact that, the reach of any cell at time $k$ is at most $2^{k}$, so that the last in the line cannot even see the order to fire in fewer than $\lceil\log n\rceil-1$ steps. To summarise it, we have shown the following.

Proposition 3 The FSSP can be solved in optimal time $\Theta(\log n)$ on a DNCA which is a line of $n$ cells.

\section{CONCLUSION AND FUTURE WORK}

We have given a basic definition of Dynamic Neighbourhood Cellular Automata and have shown that a DNCA can exhibit an exponential speed-up over a classical static neighbourhood CA. There are a number of ways to extend the model as well as many other algorithmic and complexity questions. One could consider:

1. cells that are more powerful than deterministic finite-state automata. These could be finite automata whose memory is $\Theta(\log n)$, so that a cell could know its own name or memorise another cell's name. In this case, one may need to restrict in some way the transition function $\delta$, so that a cell is not as powerful as a log-space Turing machine.

2. complexity measures other than running time. An example of this, particularly relevant to Ubiquitous Computations could be "energy", i.e. the number of times a cell receives/sends a message or changes its neighbourhood. In order to incorporate this into the formal definition, one has to make the message passing explicit so as to ensure that a cell "sleeps" (i.e. consumes no energy) if no messages have been passed onto it by its neighbours.

3. DNCA consisting of non-identical cells, i.e. cells that have different computational power. Make precise the notion of input to and output of a DNCA.

4. different algorithmic problems that have been studied elsewhere, e.g. in the context of Distributed Computing. These might include Leader Election, Byzantine Agreement etc. In order to break the symmetry, one will be forced to use randomisation and, thus, to extend the formal definition of a DNCA to allow for it.

5. creating a simple programming language for DNCA, studying its semantics and even designing and implementing some king of a formal verification tool based on these.

\section{Bibliography}

[1] Amir M. Ben-Amram. What is a "pointer machine"? SIGACT News, 26(2):88-95, June 1995.

[2] Jean-Christophe Dubacq. Different kinds of neighborhood-varying cellular automata. Maîtrise / honour bachelor degree, École normale supérieure de Lyon, 1994.

[3] Niloy Ganguly et al. A survey on cellular automata. Technical report, Centre for High Performance Computing, Dresden University of Technology, December 2003. 
[4] Jarkko Kari. Theory of cellular automata: A survey. Theoretical Computer Science, 334(13):3-33, April 2005.

[5] Jacques Mazoyer. An overview of the firing squad synchronization problem. In Christian Choffrut, editor, Automata Networks, volume 316 of Lecture Notes in Computer Science, pages 82-94. Springer, 1986.

[6] Edward F. Mooore. The firing squad synchronization problem. In Edward F. Moore, editor, Sequential Machines, Selected Papers, pages 213-214. Addison-Wesley, Reading, MA, 1964.

[7] John Von Neumann. The Theory of Self-Reproducing Automata. University of Illinois Press, 1966.

[8] Azriel Rosenfeld and Angela Y. Wu. Cellular graph automata i and ii. Information and Control, 42(3):305-353, Sep 1979.

[9] Azriel Rosenfeld and Angela Y. Wu. Reconfigurable cellular computers. Information and Control, 50(1):64-84, July 1981.

[10] Morris Sloman, et al. UKCRC grand challenges for computing research, ubiquitous computing: Science and design. Available at http://www-dse.doc.ic.ac.uk/Projects/ UbiNet/GC/index.html.

[11] Stephen Wolfram. A new kind of Science. Wolfram Media Inc., Champaign, llinois, US, United States, 2002. 